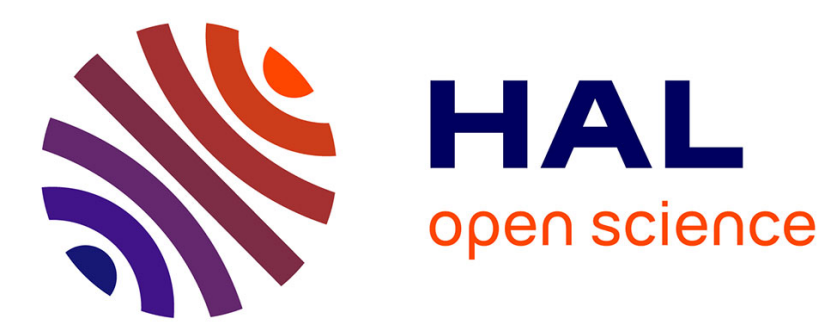

\title{
Scale Space Operators on Hierarchies of Segmentations
}

\author{
Bangalore Ravi Kiran, Jean Serra
}

\section{To cite this version:}

Bangalore Ravi Kiran, Jean Serra. Scale Space Operators on Hierarchies of Segmentations. 4th International Conference on Scale Space and Variational Methods in Computer Vision (SSVM 2013), Jun 2013, Leibnitz, Austria. pp.331-342, 10.1007/978-3-642-38267-3_28 . hal-00802447

\section{HAL Id: hal-00802447 \\ https://hal.science/hal-00802447}

Submitted on 19 Mar 2013

HAL is a multi-disciplinary open access archive for the deposit and dissemination of scientific research documents, whether they are published or not. The documents may come from teaching and research institutions in France or abroad, or from public or private research centers.
L'archive ouverte pluridisciplinaire HAL, est destinée au dépôt et à la diffusion de documents scientifiques de niveau recherche, publiés ou non, émanant des établissements d'enseignement et de recherche français ou étrangers, des laboratoires publics ou privés. 


\title{
Scale Space Operators on Hierarchies of Segmentations
}

\author{
B. Ravi Kiran, Jean Serra \\ Université Paris-Est, Laboratoire d'Informatique Gaspard-Monge, A3SI, ESIEE \\ \{kiranr, j.serra@esiee.fr
}

\begin{abstract}
A hierarchy of segmentations(partitions) is a multiscale set representation of the image. This paper introduces a new set of scale space operators or transformations on the space of hierarchies of partitions. An ordering of hierarchies is proposed which is endowed by an $\omega$-ordering based on a global energy over the classes of the hierarchy. A class of Matheron semigroups are shown to exists in this ordering of hierarchies. A second contribution is the saliency transformation which fuses a saliency function corresponding to a hierarchy, with an external function, rendering a new or transformed saliency function. The results are demonstrated on the Berkeley dataset.
\end{abstract}

\section{Introduction}

This paper addresses the questions of synthesizing and improving hierarchies of segmentations by means of scale space operators. A hierarchy of partitions has been previously obtained, and is given. It provides a stack of coarser and coarser segmentations of the scene under study. Some external information, or "ground truth" composed of sets, drawings, auxiliary numerical functions, etc.. , may come, or not, with the hierarchy. The problem is thus twofold, and suggests to separate the situations with no external information form those with ground truth. They lead indeed to two rather different approaches

The first one -no outside information- is based on already known techniques which extract an optimal cut form the hierarchy by minimizing some energy $\omega$. The most often, the energy $\omega$ depends on a positive parameter [11] [4] [13] [5]. Under which conditions this parameter can be understood as a space scaler, leading to an improved hierarchy and to scale space semi-groups? This will be the matter of section 3 , which is preceded by a reminder on optimal cuts in hierarchies.

The second situation involves disparate data. For answering the question "How to enrich the hierarchy with ground truths?" we have to find a common basis to express them, and from this basis, to build up a few laws of composition. The scale spacing will then intervene as distance functions associated with the ground-truths. These questions will be treated in sections 4 . 

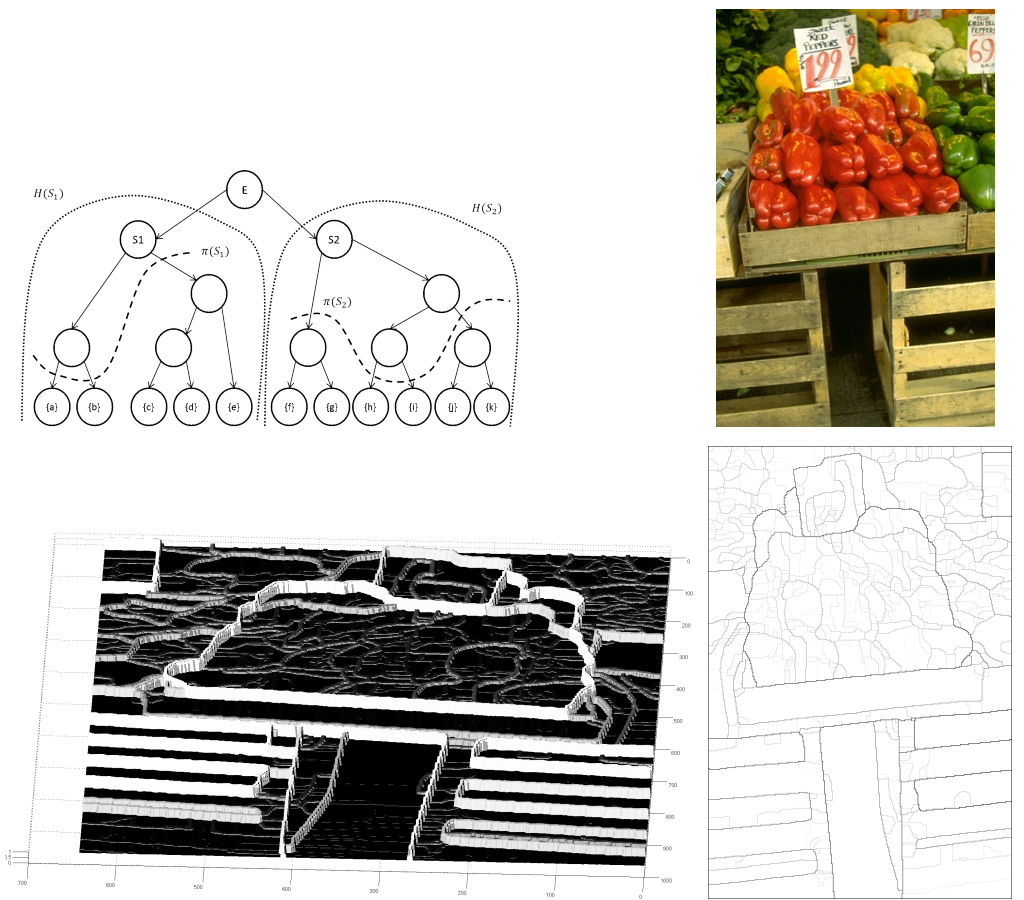

Fig. 1. Top: Dendrogram representation of hierarchy, Input 25098 Image, Bottom: Topographic view of UCM, Inverted (and contrasted for better view) Ultrametric contour Map(UCM) where the edges with strongest saliency values are the darkest, and the weakest values are the lowest, while zeros are white(background).

\section{Optima cuts and hierarchies (reminder)}

The definitions and prerequisites needed in understanding the rest of the paper are given in this section [5], [12]. The usual distinction between continuous and digital spaces is not appropriate for the general theory developed in sections 2 to 4 . What is actually needed reduces to the two following assumptions

i) the space $E$ to partition is topological, like $\mathbb{R}^{2}, \mathbb{Z}^{2}$, or others,

ii) the smallest partition $\pi_{0}$ taken into account has a finite number of classes.

The first assumption allows us to speak of frontiers between classes, or edges. The second one aims to avoid things like fractal sets.

\subsection{Partitions, partial partitions}

Intuitively, a partition of $E$ is a division of this set into classes, i.e. regions that do not overlap, and whose union gives $E$. Below, the symbols $S, T$ stand for 
classes, and $\pi$ for partitions. Partition $\pi_{1}$ is smaller than partition $\pi_{2}$ when each class of $\pi_{1}$ is included in a class of $\pi_{2}$. This condition provides an ordering on the partitions, called refinement, which in turn induces a complete lattice.

Let $S$ be a subset of $E$. Following Ch. Ronse [10], any partition $\pi(S)$ of $S$ is called partial partition of support $S$ (in short p.p.). In particular, the partial partition of $S$ into a single class is denoted by $\{S\}$. If the $q$ classes of the partition $\pi(S)$ are $\left\{T_{u}, 1 \leq u \leq q\right\}$, one writes

$$
\pi(S)=T_{1} \sqcup . . T_{u} . . \sqcup T_{q},
$$

where the symbol $\sqcup$ indicates that the classes are concatenated. The set of all partial partitions of $E$ is denoted by $\mathcal{D}$.

An energy on $\mathcal{D}$ is a numerical function $\omega: \mathcal{D} \rightarrow[0, \infty]$. In the following, $\mathcal{D}$ will be provided with several energies $\omega$, which may satisfy two axioms

i) $\omega$ is $h$-increasing, i.e.

$$
\omega\left(\pi_{1}\right) \leq \omega\left(\pi_{2}\right) \quad \Rightarrow \quad \omega\left(\pi_{1} \sqcup \pi_{0}\right) \leq \omega\left(\pi_{2} \sqcup \pi_{0}\right) .
$$

where $\pi_{1}$ and $\pi_{2}$ are two partial partitions of same support, and $\pi_{0}$ a partial partition disjoint from $\pi_{1}$ and $\pi_{2}$,

ii) $\omega$ is singular, when the energy $\omega(\{S\})$ of class $S$ is differs from that of any p.p. of $S$, i.e.

$$
\pi(S) \text { p.p. of }\{S\} \quad \Rightarrow \omega(\{S\}) \neq \omega(\pi(S)) .
$$

The geometrical meaning of Rel.(1) is depicted in Figure 2.

\subsection{Hierarchies of partitions}

A hierarchy $H$ is a chain of ordered partitions $\pi_{i}$, i.e.

$$
H=\left\{\pi_{i}, 0 \leq i \leq n \mid i \leq k \leq n \Rightarrow \pi_{i} \leq \pi_{k}\right\}
$$

where $\pi_{n}$ is the partition $\{E\}$ of $E$ in a single class called the root. The classes of the finest partition $\pi_{0}$ are called the leaves, and the intermediary classes are the nodes.

Let $S_{i}(x)$ be the class of partition $\pi_{i}$ of $H$ at point $x \in E$. Denote by $\mathcal{S}$ the set of all classes $S_{i}(x)$ of $H$, i.e. $\mathcal{S}=\left\{S_{i}(x), x \in E, 0 \leq i \leq n\right\}$. Expression (3) means that at each leaf $x$ the family of those classes $S_{i}(x)$ of $\mathcal{S}$ that contain $x$ forms a finite chain $\mathcal{S}_{x}$ in $\mathcal{P}(E)$, of nested elements from $S_{0}(x)$ to $E$ :

$$
\mathcal{S}_{x}=\left\{S_{i}(x), 0 \leq i \leq n\right\} .
$$

According to a classical result, a family $\left\{S_{i}(x), x \in E, 0 \leq i \leq n\right\}$ of indexed sets generates the classes of a hierarchy iff

$$
x, y \in E \Rightarrow S_{i}(x) \subseteq S_{j}(y) \text { or } S_{i}(x) \supseteq S_{j}(y) \text { or } S_{i}(x) \cap S_{j}(y)=\varnothing .
$$




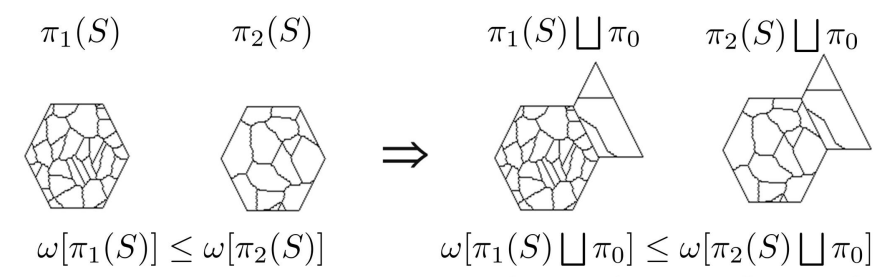

Fig. 2. $h$-increasingness

The partitions of a hierarchy may be represented by their classes, or by the saliency map of the edges, or again by a dendrogram where each node of bifurcation is a class $S$, as depicted in Figure 1. The classes of $\pi_{i-1}$ at level $i-1$ which are included in class $S_{i}(x)$ are said to be the sons of $S_{i}(x)$. The set of all classes $S$ of all partitions involved in $H$ is denoted by $\mathcal{S}(H)$. Clearly, the descendants of each $S$ form in turn a hierarchy $H(S)$ of root $S$, which is included in the complete hierarchy $H=H(E)$.

\subsection{Cuts in a hierarchy}

Any partition $\pi$ of $E$ whose classes are taken in $\mathcal{S}$ defines a cut $\pi$ in a hierarchy $H$. The set of all cuts of $E$ is denoted by $\Pi(E)=\Pi$. Every "horizontal" section $\pi_{i}(H)$ at level $i$ is obviously a cut, but several levels can cooperate in a same cut, such as $\pi\left(S_{1}\right)$ and $\pi\left(S_{2}\right)$, drawn with thick dotted lines in Figure 1. Similarly, the partition $\pi\left(S_{1}\right) \sqcup \pi\left(S_{2}\right)$ of the figure generates a cut of $H(E)$.

Given an energy $\omega$ over the set $\mathcal{D}(E)$ of the partial partitions of $E$, an optimal cut $\pi^{*} \in \Pi(E)$ is a cut that minimizes $\omega$, i.e. such that $\omega\left(\pi^{*}\right)=\inf \{\omega(\pi) \mid$ $\pi \in \Pi(E)\}$. Now, though the hierarchies are discrete, the number of their possible cuts becomes rapidly huge: a small hierarchy of 200 leaves and 10 levels generates billions of cuts! How to find out the best one? The following two theorems answer the question

Theorem 1. Let $H$ be a hierarchy and $\omega$ be a h-increasing and singular energy. Energy $\omega$ induces an ordering on the set $\Pi(E)$ of all cuts of $H$. Given two cuts $\pi, \pi^{\prime} \in \Pi(E)$, cut $\pi$ is said to be less energetic than cut $\pi^{\prime}$ w.r.t. $\omega$, and one writes $\pi \leq_{\omega} \pi^{\prime}$, when in each class $S$ of the refinement supremum $\pi \vee \pi^{\prime}$ the p.p. of $\pi$ inside $S$ is less energetic than that of $\pi^{\prime}$ inside $S$. The energetic ordering induces the $\omega$-lattice $\left(\wedge_{\omega}, \vee_{\omega}\right)$.

In the notation, we distinguish the refinement lattice from the $\omega$-lattice by using for the former the three symbols $\leq, \vee$, and $\wedge$, without $\omega$ subscript. The meaning of the energetic lattice $\left(\wedge_{\omega}, \vee_{\omega}\right)$ is clear: it associates energetic minimum and maximum with each class of $\pi \vee \pi^{\prime}$, and not globally only.

Theorem 2. Let $\omega$ be h-increasing and singular energy. Then for any $H \in \mathcal{H}$ and any node $S$ of $H$ with $p$ sons $T_{1} . . T_{p}$ of optimal cuts $\pi_{1}^{*}, . . \pi_{p}^{*}$, there exists a 


\begin{tabular}{|l|l|}
\hline 10 & 5 \\
\hline 10 & 5 \\
\hline
\end{tabular}

$a$

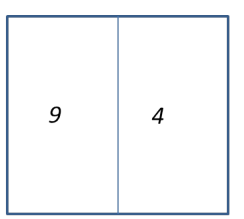

$b$

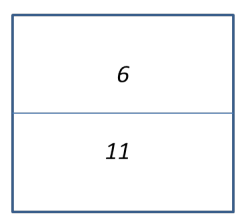

$c$

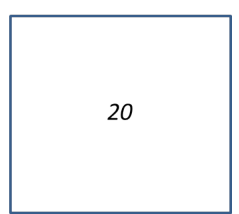

$d$

Fig. 3. The leaves are the four classes of $a$. The three levels of the hierarchy $H_{1}$ are [a b d] and those $H_{2}$ ) are [a c d], and $d$ is the whole space. The indicated energies $\omega$ show that $H_{1} \leq_{\omega} H_{2}$.

unique optimal cut of the sub-hierarchy of root $S$. It is either the cut $\pi_{1}^{*} \sqcup \pi_{2}^{*} . . \sqcup \pi_{p}^{*}$, or the one class partition $\{S\}$ itself:

$$
\omega\left(\pi^{*}(S)\right)=\min \left\{\omega(\{S\}), \omega\left(\pi_{1}^{*} \sqcup \pi_{2}^{*} . . \sqcup \pi_{p}^{*}\right)\right\}
$$

Theorem 2 governs the choices of models for energies, and their implementations:

Firstly, the dynamic programming Rel.(5) allows us to find the optimal cut of $H$ in one ascending pass. The nodes of $H$ above the leaves have to be visited according to an order which respects the inclusions. One then compares the energy of each node with that of the p.p. of its sons, and the less energetic of the two is kept for continuing the ascending pass, and so on until the top node $E$ is reached [4], [5].

Secondly, the obtained optimal cut $\pi^{*}(E)$ is indeed globally less energetic than any other cut in $H$, but, moreover, if we compare $\pi^{*}$ with any other partition $\pi$ of $E$, then in each class $S$ of the refinement supremum $\pi^{*} \vee \pi$ the energy of $\pi^{*}$ is smaller than that of $\pi$.

\section{Openings on $\mathcal{H}(\mathcal{S})$}

Studies on hierarchies often hold on the family of all hierarchies whose nodes are taken among the set $\mathcal{S}$ of nodes of some initial hierarchy $H$, a family denoted by $\mathcal{H}(\mathcal{S})$ below. Now, optimal cutting is an operation which maps hierarchies on partitions. If we wish to insert it in a series of transformations on hierarchies, this optimal cutting must be interpreted differently.

We observe firstly that both energetic and refinement orderings on partial partitions induce orderings on the set $\mathcal{H}(\mathcal{S})$ of hierarchies, for which $H_{1} \leq H_{2}$ when at any level $i, \pi_{1}(i) \leq \pi_{2}(i)\left(\operatorname{resp} . \pi_{1}(i) \leq_{\omega} \pi_{2}(i)\right)$. For the refinement one, the optimal element is the cylindric hierarchy whose all horizontal sections are the leaves partition, and the maximal one is obtained by taking the one class partition $\{E\}$ at all levels, leaves level excepted. In the $\omega$-lattice, the two extreme elements are the two cylinders $H^{*}$ and $H^{* *}$ whose all sections above the leaves level are the optimal cut, or the maximal one. 


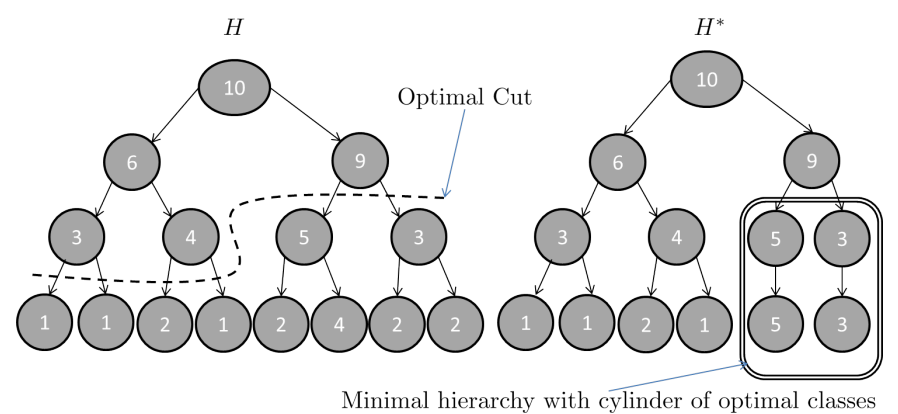

Fig. 4. Minimal pyramid $H^{*}$ obtained by replacing non optimal classes in $H$ up till level of the optimal cut

Consider now the refinement supremum $H \vee H^{*}$ of $H$ and of the $\omega$ - optimal cylinder $H^{*}$ and view it as an element of the $\omega$-lattice $\mathcal{H}(\mathcal{S})$.

Theorem 3. The operation $\gamma_{\omega}^{*}(H)=H \vee H^{*}$ from the $\omega$-lattice $\mathcal{H}(\mathcal{S})$ into itself is an opening.

Proof. $\gamma_{\omega}^{*}$ is anti-extensive, since each class $S$ of $H$ is replaced by a less energetic class of $H^{*}$ when $S \leq S^{*}$ and left unchanged when not. On the other hand $\gamma_{\omega}^{*}\left[\gamma_{\omega}(H)\right]=H \vee H^{*} \vee H^{*}=\gamma_{\omega}^{*}(H)$, which is thus idempotent. Finally, $\gamma_{\omega}^{*}$ is also increasing since when $H \leq_{\omega} H^{\prime}$ then each class of $H \vee H^{*}$ has an energy smaller or equal to that of the class of same level in $H \vee H^{\prime *}$, which achieves the proof.

Introduce the cone $\mathcal{S}(x)=\left\{S_{i}(x), 1 \leq i \leq N\right\}$ of all classes of $H$ that contain the leaf $x$. As $x$ spans $\pi_{0}$, the cones $\left\{\mathcal{S}(x), x \in \pi_{0}\right\}$ characterize the hierarchy $H$. The transform $\gamma_{\omega}^{*}(H)$ can be described by its characteristic cones $\mathcal{S}^{*}(x)$ :

$$
\begin{array}{ll}
\mathcal{S}^{*}(x)=\left\{S_{j}^{*}(x)=S_{i}^{*}(x),\right. & 1 \leq j \leq i\} \\
\mathcal{S}^{*}(x)=\left\{S_{j}^{*}(x)=S_{i}(x),\right. & i<j \leq N\},
\end{array}
$$

where $S_{i}^{*}(x)$ denotes the class of the optimal cut at leaf $x$, and $i$ the level at which this class is located. In the cone $\mathcal{S}^{*}(x)$ all classes below level $i+1$ are replaced by $S_{i}^{*}(x)$, and the other ones are those of $H$ itself.

Instead of $H \vee H^{*}$, we can as well start from $H \wedge H^{*}$, and consider the operation $\zeta_{\omega}^{*}(H)=H \wedge H^{*}$, which also turns out to be an opening. In the cone at leaf $x$ of $\zeta_{\omega}^{*}(H)$ all classes above level $i+1$ are replaced by $S_{i}^{*}(x)$, and the other ones are those of $H$ itself.

\subsection{Semi-groups of climbing energies on $\mathcal{H}(\mathcal{S})$}

We now consider a climbing family $\{\omega(\lambda), \lambda \in \Lambda\}$ of energies, i.e. a family of $h$-increasing and single energies, as previously, to which we add the axiom of 
scale increasingness [5]. This axiom states that if the energy $\omega(\lambda ; S)$ of node is lesser than the energies $\omega(\lambda ; \pi)$ for all p.p. $\pi$ of support $S$, then the inequality remains true for the energies $\omega(\mu), \lambda \leq \mu$ :

$$
\lambda \leq \mu \text { and } \omega(\lambda ; S) \leq \omega(\lambda ; \pi) \Rightarrow \omega(\mu ; S) \leq \omega(\mu ; \pi), \quad S \in \mathcal{S} .
$$

The climbing family $\{\omega(\lambda), \lambda \in \Lambda\}$ generates a semi-group of operators. Denote by $H_{\lambda}^{*}$ and $H_{\mu}^{*}$ the smallest elements of $\mathcal{H}(\mathcal{S})$ for the two $\omega(\lambda)$-lattice and $\omega(\mu)$-lattice respectively. The scale increasingness Rel.(6) implies that $H_{\lambda}^{*} \leq H_{\mu}^{*}$, or equivalently:

$$
H_{\lambda}^{*} \vee H_{\mu}^{*}=H_{\mu}^{*} \quad H_{\lambda}^{*} \wedge H_{\mu}^{*}=H_{\lambda}^{*}
$$

for the refinement supremum and infimum. It follows that:

$$
\gamma_{\omega(\mu)}^{*}\left[\gamma_{\omega(\lambda)}^{*}(H)\right]=\left(H \vee H_{\lambda}^{*}\right) \vee H_{\mu}^{*}=\gamma_{\omega(\mu)}^{*}(H) .
$$

As the two suprema commute, the optimal cut openings $\gamma_{\omega}^{*}$ turn out to satisfy the Matheron semi-group ${ }^{1}$ :

$$
\gamma_{\omega(\lambda)}^{*} \circ \gamma_{\omega(\mu)}^{*}=\gamma_{\omega(\mu)}^{*} \circ \gamma_{\omega(\lambda)}^{*}=\gamma_{\max \{\omega(\lambda), \omega(\mu)\}}^{*} \quad \lambda, \mu>0 .
$$

Concerning the dual form $\zeta_{\omega}^{*}$ one finds similarly/

$$
\zeta_{\omega(\lambda)}^{*} \circ \zeta_{\omega(\mu)}^{*}=\zeta_{\omega(\mu)}^{*} \circ \zeta_{\omega(\lambda)}^{*}=\zeta_{\min \{\omega(\lambda), \omega(\mu)\}}^{*} \quad \lambda, \mu>0 .
$$

This time, the lower energy imposes its law. Finally, the whole collection of the optimal cuts can appear in the synthetic hierarchy

$$
H_{s y n}=\left(\ldots\left(\left(H \vee_{\omega 1} H_{\lambda 1}^{*}\right) \vee_{\omega 2} H_{\lambda 2}^{*}\right) \ldots\right) \vee_{\omega p} H_{\lambda p}^{*}
$$

which is a succession of the increasing optimal cuts of the energies $\omega 1, \omega 2, \ldots \omega p$.

\section{Saliency transformation}

We now address the second question set in the introduction: how to merge hierarchy and ground-truth ? This time, hierarchy $H$ is represented by its saliency; i.e. by a weighting function associated with the edges between classes of $H$ [8]. For a given edge, this function, constant along the edge, is the level of $H$ when the edge disappears. If we associate also one or more numerical functions $g$ with the ground-truth, the merging question comes back to that of combining numerical functions for generating a new saliency.

In order to make saliencies and hierarchies equivalent notions, we consider the latter as sequences of partitions that appear at different levels, and not

\footnotetext{
1 There are two broad classifications of scale spaces semigroups based on the underlying algebraic structure, used in scale space applications. First is the linear semigroup, based on a vector space. Second is the semigroup of Matheron's granulometries [7] which uses an underlying lattice for analysis, and where the most active transformation imposes its law.
} 


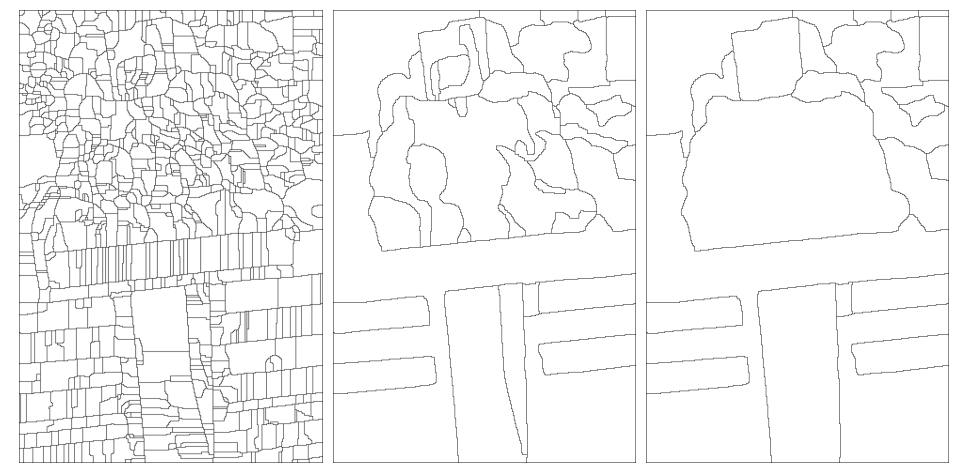

Fig. 5. A set of Optimal cuts form a Matheron semigroup : Three partitions of 25098 Image at $\lambda=0$ (leaves), 5000 and 8000

just ordered. Any strictly increasing mapping $\alpha$ of the levels, e.g. square root, log, etc., transforms a saliency into another one, as well as the addition by a constant value. However, a distribution of arbitrary weights on the edges may not be saliency. It is also required that by removing one edge one still maintains a partition, i.e. that one does not create pending edges. This condition is formalized below by the operation of class opening.

\subsection{The class opening}

This operation appeared in literature on the same date, in two independent contexts. The first is the ultrametric opening [6] which concerns discrete classifications by ultrametrics. The second is the pruning [14], which is a morphological thinning, and transforms a skeleton into a skeleton by zones of influence. More recently, in [9] the same opening allows to identify hierarchical segmentation with ultrametric watershed in digital spaces (see also [3]). Here, we start from the same notion, but more simply, without any ultrametric, or graphs or any digital background.

The difference between what follows and the three above references concerns the consequences of the class opening, namely the corollary 1 , and above all the key theorem of structure 4, ignored in [6], [14], [9], and which answers the question set in the first sentence of this section. Given a finite set $E$ of simple arcs in the $2-D$ space $\mathbb{R}^{2}$ or $\mathbb{Z}^{2}$, which can meet at their extremities only, consider the binary operation $\gamma: \mathcal{P}(E) \rightarrow \mathcal{P}(E)$ which reduces each set of arcs $X \in \mathcal{P}(E)$ to the closed contours it may produce.

Theorem 4. the operation $\gamma: \mathcal{P}(E) \rightarrow \mathcal{P}(E)$ is an opening.

Proof. Let be $X, Y \in \mathcal{P}(E)$. Then each closed contour of $X$ is also a closed contour of $Y$, and $\gamma(X) \subseteq \gamma(Y)$. On the other hand, as $\gamma(X)$ is reduced to its contours, $\gamma \gamma(X)=\gamma(X)$. Finally, $\gamma(X) \subseteq X$, which achieves the proof. 


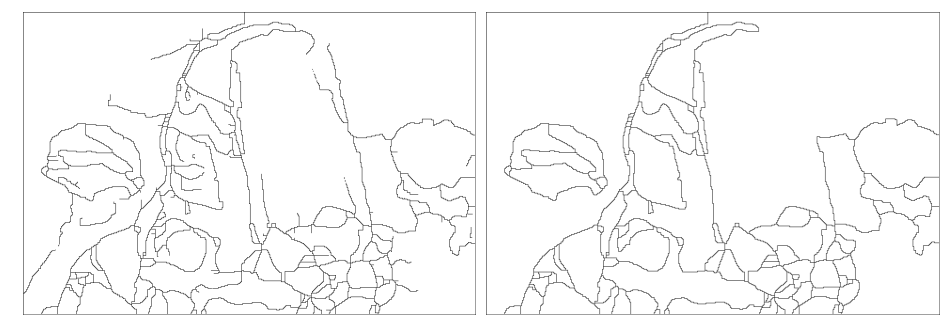

Fig. 6. A class opening demonstrated: Initial set of arcs, Class opening providing a partition

We call " binary class opening" the operation $\gamma$, since it selects the arcs that delineate the classes of a partition of $E$.

The numerical extension of $\gamma$, for which we keep the same symbol $\gamma$, holds on a numerical function $g$ on the $2-D$ underlying space $\mathbb{R}^{2}$ or $\mathbb{Z}^{2}$. The edges of the leaves are thus formed by elements of $E$, points or pixels. Denote by $X_{t}(g)$ the set of pixels of the leaves where $g$ is $\geq t$, and define the numerical opening $\gamma(g)$ by its level sets $X_{t}[\gamma(g)]$ by putting

$$
X_{t}[\gamma(g)]=\gamma\left[X_{t}(g)\right], \quad t>0
$$

As the number of edges is finite, the number of changes between level sets is also finite. Let $S_{i+1}$ be a class which appears at level $t_{i+1}$. When $t$ decreases, the next new class $S_{i}$ appears at $t_{i}$. Since there is no change in the interval ] $\left.t_{i}, t_{i+1}\right]$, we have

$$
t_{i}=\inf \left\{g(x) \mid x \in \partial S_{i}\right\}
$$

We assume that $g$ is discrete, or lower semi-continuous, so that the value $t_{i}$ occurs at one point of some edge $e_{i}$ of $S_{i}$. This value is nothing but the weight of the edge $e_{i}$ in the saliency transform $\gamma(g)$ which in turn generates hierarchy $H$, and $t_{i}$ is the highest level of class $S_{i}$ in $H$. If several classes appear at $t_{i}$, generated by several closing edges, then their intersections are empty and the description remains valid. Therefore, an opening being characterized by its invariants, we can state

Corollary 1. Let $\mathcal{G}$ be the family of all integer functions $g: \mathbb{R}^{2} \rightarrow \mathbb{Z}^{+}$, or $\mathbb{Z}^{2} \rightarrow \mathbb{Z}^{+}$. The image $\mathcal{I}=\gamma(\mathcal{G})$ of $\mathcal{G}$ under the class opening $\gamma$ is exactly the family of all possible saliencies on the set $E$ of the leaves edges.

\subsection{Composition of class openings}

The composition problems are the following:

1- A first saliency, $s$ say, already weights the set of edges $E$. When a non negative function $g$ over space the underlying space $\mathbb{R}^{2}$ or $\mathbb{Z}^{2}$ is introduced, how to compose it with $s$ ? 
2- When in turn a second function, $g_{2}$, acts on the saliency $s_{1}$ resulting of $g_{1}$, how the two effects are composed?

The combination of saliencies and functions is not straightforward. Given $s$ and $g$, the sum, the difference, the product, the ratio, the supremum, or the infimum between $s$ and $g$, may not be saliencies. The only exception arises when both $s$ and $g$ are saliencies. Then their supremum results in a saliency, but not the other operations. However, a few nice properties can be stated:

Theorem 5. Let $g_{1}$ and $g_{2}$ be two non negative functions on $\mathbb{R}^{2}$ or $\mathbb{Z}^{2}$, then:

i) $\gamma\left(g_{1}\right)$ (resp. $\gamma\left(g_{2}\right)$ ) is the largest saliency smaller than $g_{1}$ (resp. $\left.g_{2}\right)$;

ii) $\gamma\left(g_{1}\right) \vee \gamma\left(g_{2}\right)$ is the largest saliency whose value at each edge is smaller or equal to that of $\gamma\left(g_{1}\right)$ or $\gamma\left(g_{2}\right)$;

iii) if $g_{1} \circledast g_{2}$ denotes an operation from $\mathcal{G} \times \mathcal{G} \rightarrow \mathcal{G}$, such as,,$+- \times, \div, \vee$,or $\wedge$, then $\gamma\left(g_{1} \circledast g_{2}\right)$ is the largest saliency smaller than $g_{1} \circledast g_{2}$, and $\gamma\left(g_{1} \vee g_{2}\right) \leq$ $\gamma\left(g_{1}+g_{2}\right)$.

In all cases the resulting saliency is unique.

The proposition suggests two paths for combining saliencies. Given a primary saliency $s$ and the ground truths $g_{1}, g_{2}, \ldots g_{n}$, the sequence $s, s \vee \gamma\left(g_{1}\right), s \vee \gamma\left(g_{1}\right) \vee$ $\gamma\left(g_{2}\right)$, etc..provides an increasing family of saliencies, and the ground truths commute in the various $s \vee \gamma\left(g_{1}\right) \vee \ldots \gamma\left(g_{i}\right)$. Alternative families are given when we compose various $g_{i}$ and then perform the class opening, namely $\gamma\left(s \vee g_{1}\right)$, $\gamma\left(s \vee g_{1} \vee g_{2}\right)$, etc.. and $\gamma\left(s+g_{1}\right), \gamma\left(s+g_{1}+g_{2}\right)$, etc..In all cases the series is increasing, and simplify more the hierarchy $H(s)$ when suprema are involved.

Owing to the equivalence "saliencies $\Leftrightarrow$ hierarchies" all the above compositions map the whole space $\mathcal{H}$ of the hierarchies into itself. We have the succession

$$
H \rightarrow \text { saliency } s \rightarrow \text { saliency } \gamma(s, g) \rightarrow \text { new hierarchy } H^{\prime}
$$

We are no longer in the situation of the semi-groups of section 3, where the framework was restricted to $\mathcal{H}(\mathcal{S})$. Here new classes, absent in $H$, can appear in $H^{\prime}$. The adopted approach, via the class opening, provides also the space $\mathcal{H}$ with a lattice structure isomorphic to that of the openings.

\section{$5 \quad$ Experiments and analysis}

Here we demonstrate an example of the class opening on the Ultrametric contour map (UCM) from the Berkeley database [1].

\subsection{Saliency transformation by ground truth}

Conventionally the ground truth information is intended to assess the quality of a segmentation, here a hierarchy $H$ of segmentations. Here in the place of evaluating the hierarchy, we analyse it with respect to the given ground 

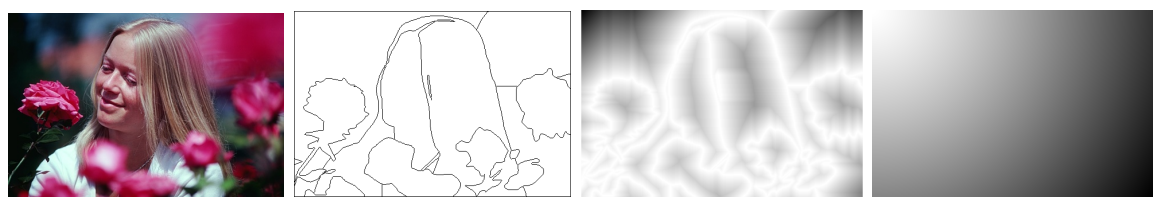

Fig. 7. 239096 Image, One of the Ground truth partitions $\left(G_{1}\right)$, Inverse distance function for $g_{1}$, Point ground truth inverse distance function $g_{p}$ (point at top right), where $g_{1}$ and $g_{p}$ are the corresponding euclidean distance functions
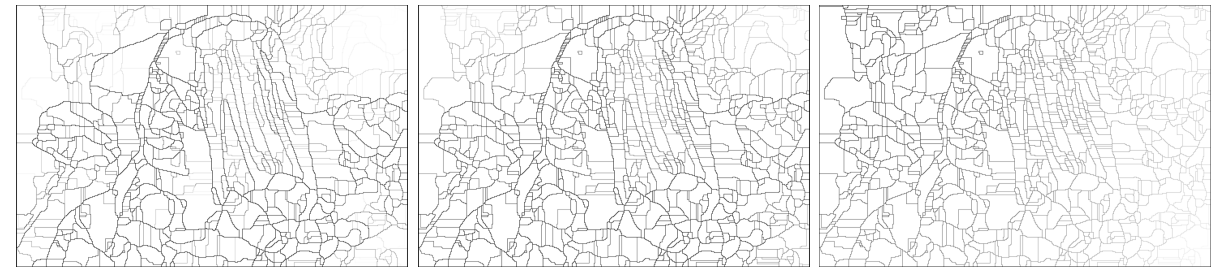

Fig. 8. Original Saliency $s$ (Image 239096), new transformed saliency by class opening $\gamma\left(g_{1}+s\right)$ with ground truth $G_{1}$. Saliency by class opening with point ground truth $\gamma\left(g_{p}+s\right)$ to demonstrate the effect of the inverse distance function. we see the profile of the transformed saliency $\gamma\left(g_{p}+s\right)$ follows the inverse distance function $g_{p}$

truth. The saliency transformation by a ground truth is an amelioration of the partitions in the hierarchy to generate new partitions with the same edges ordered by combined effect of: 1 . proximity to the ground truth 2 . high saliency. More clearly, how do we combine a ground truth and a hierarchy of partitions?

The inputs given to us are the saliency function $s$ representing the initial hierarchy $H$ and the ground truth partition of edges $G$. Here we use the distance function of ground truth $d$, to define the inverse distance function $g=1-d$. The output is a new saliency $\gamma(s+g)$ and thus a new hierarchy $H_{g}$ which contains partial partitions from $H$ that are closest in distance to the ground truth partition $G$ and the saliency (see figure 7 ).

Figure 8 summarizes the input and output saliencies. The input saliency is shown for input image 239096 from the Berkeley database. The ground truth $G_{1}$ is more or less representative of the image structure in the saliency $s$, and thus the resulting transformed saliency $s_{G_{1}}$ is not too different, except that in general edges very far from the ground truth are reduced or weakened, while the ones in close proximity are reinforced. For the sake of pedagogy we demonstrate with a inverse distance function of a point shown in Figure $7\left(g_{p}\right)$ and its corresponding saliency $\gamma\left(g_{p}+s\right)$. We see the radial attenuation in the transformed saliency. 


\section{6 conclusion}

This paper discussed two main contributions, namely: 1 . The different scale space semigroups on hierarchies of partitions. 2. A saliency transform that introduces external information into some initial hierarchy. The synthesis was obtained by means of a class opening that reduces a set of arcs containing loops into just its loops, and its numerical equivalent. An application of fusing the ground truth and saliency function was demonstrated, which reordered arcs in the hierarchy based jointly on the saliency and ground truth proximity. The distance function here can be replaced by other external information, like color and depth information, [2], thus enabling the evaluation of the hierarchy using many different functions. Following this algebraic structure, applications in multi-variable fusion and feature extraction will be explored.

Acknowledgements The authors are grateful to Prof. L. Najman for his valuable comments on the class opening.

\section{References}

1. Arbeláez, P., Maire, M., Fowlkes, C., Malik, J.: Contour Detection and Hierarchical Image Segmentation. IEEE PAMI 33 (2011)

2. Calderero F., Marques F., Hierarchical fusion of color and depth information at partition level by cooperative region merging, ICASSP '09 Proceedings

3. Cousty J., Najman L., Serra J., Raising in Watershed Lattices, 2008 IEEE Intern. Conf. on Image Processing, ICIP 2008, San Diego 13 -17 Oct. 2008.

4. Guigues L., Cocquerez J.P., Le Men H., Scale-Sets Image Analysis, Int. Journal of Computer Vision 68(3), 289-317, 2006.

5. Kiran B.R. and Serra J., Global-Local optimization on hierarchies, Pattern Recognition Letters, special issue 2013.

6. Leclerc B., Description combinatoire des ultramétriques, Mathématiques et Sciences Humaines vol. 73, pp. 5-37, 1981

7. Matheron G., Random sets and integral geometry, John Wiley and Sons, 1975, ISBN 978-0-471-57621-1.

8. Najman, L., Schmitt, M. Geodesic saliency of watershed contours and hierarchical segmentation PAMI, IEEE transactions on, 1996

9. Najman, L., On the equivalence between hierarchical segmentations and ultrametric watersheds, JMIV 40(3),231-247, 2011

10. Ronse C., Partial Partitions, Partial Connections and Connective Segmentation. JMIV 32(2): 97-125 (2008)

11. Salembier P., Garrido L., Binary Partition Tree as an Efficient Representation for Image Processing, Segmentation, and Information Retrieval. IEEE Trans. on Image Processing, 2000, 9(4): 561-576.

12. Serra, J., Hierarchy and Optima, in Discrete Geometry for Computer Imagery 2011, LNCS 6007, Springer, pp 35-46

13. Serra J., Kiran B.R., Cousty J., Hierarchies and Climbing Energies. CIARP 2012,: LNCS 7441, 821-828

14. Serra J., Image Analysis and Mathematical Morphology, Page 397. Academic Press 1983. ISBN:0126372403 